\title{
FACTORES DE RIESGO SOBRE LA MORBIMORTALIDAD MATERNA Y EN MENORES DE 5 AÑOS EN EL CENTRO DE SALUD BORBÓN DE LA CIUDAD DE ESMERALDAS-ECUADOR, DURANTE EL AÑO 1994
}

\author{
M. Teresa Torres Rodriguez* \\ M. Mónica Moreno Cevallos* \\ * Licenciadas egresadas de la Pontificia Universidad Católica del Ecuador sede en Esmeraldas
}

\section{RESUMEN}

Se realizó un estudio retrospectivo, analítico, durante el año de 1994, cuyos objetivos eran los de conocer e identificar los factores de riesgo más significativos y establecer si las prácticas y creencias comunitarias implicaban probabilidad de morbimortalidad materno e infantil. El estudio se lo realizó en las áreas de salud de la comunidad Borbón de la ciudad de Esmeraldas-Ecuador, se diseñó una encuesta dirigida a las embarazadas, otra a las madres de niños vivos o muertos en el último año, validada a través de una prueba piloto. Se aplicó la encuesta sobre una muestra aleatoria de 200 niños menores de 5 años, 83 embarazadas y un grupo de 7 madres con hijos muertos por causas hipotéticas.

Palabras clave: enfermeria materno infantil, antropologia clinica.

FACTORS OF RISK ON THE MATERNAL MORBIMORTALIDAD AND IN MINORS OF 5 YEARS IN THE BORBÓN HEALTH CENTER (ESMERALDAS-ECUADOR), DURING 1994

\section{SUMMARY}

A retrospective, analytical study was made, during the year 1994. Our objectives were those to know and to identify the factors of risk more sig- nificant and establishing if the communitarian practices and beliefs implied probability of maternal and infantile morbimortalidad. The study was made it in the areas of health of Borbón community in the city of Emerald-Ecuador, designed a survey directed to the pregnant women, another one to the mothers of children alive or died in the last year, validated through a test pilot. The survey was applied on a random sample of 200 children smaller of 5 years, 83 embarrassed and a group of 7 mothers with children died by hypothetical causes.

Keywords: nursing maternal-infantile, clinical anthropology.

\section{INTRODUCCIÓN}

La morbimortalidad materna e infantil, constituye aún un problema social y de salud en el Ecuador y en toda América Latina. Las complicaciones del embarazo, parto, puerperio y abortos, determinaron las primeras causas de defunción de las mujeres en edad fértil, relacionándose a su vez esta situación con la morbilidad y mortalidad infantil por causas evitables.

La mortalidad infantil en menores de $l$ año es superior a la de los niños del grupo de 1 a 14 , debido a enfermedades de fácil prevención como las infecciones diarreicas agudas, infecciones respiratorias agudas, hipóxias neonatales, bajo peso al 
nacer, desnutrición, etc. Situación que se ha tornado muy difícil en las zonas urbanomarginales y rurales entre otras causas por la falta de infraestructura básica sanitaria, desconocimiento de prácticas para el autocuidado, además por la inaccesibilidad a los servicios de salud existente.

Es así, que los objetivos de la OMS, entre otras cosas, se establecieron estrategias, como la optimizar la atención Materno Infantil, la cual se fundamenta en el hecho de que América Latina tiene altas tasas de morbimortalidad en dichos grupos, elevadas tasas de crecimiento de la población reflejada en el panorama demográfico y en los factores propios del medio donde transcurre el proceso. (6)

El Ministerio de Salud Pública del Ecuador, ha implementado acciones tendientes en mejorar la situación materno infantil, sin embargo la falta de una política definida a nivel nacional así como la insuficiente coordinación con otros organismos el presupuesto limitado destinado a otros programas y la escasez de recursos humanos plenamente capacitados, hacen que el objetivo propuesto sea inalcanzable, colocando los resultados muy por debajo de las metas trazadas, cuando apenas nos encontramos a poco tiempo de llegar al año 2000. En el ámbito nacional el promedio de mortalidad materna alcanza 153 muertes por cada 100.000 nacidos vivos, siendo el Ecuador uno de los países con alta mortalidad entre los países de América Latina y el Caribe. En el Ecuador la mortalidad materna ha disminuido en los últimos años, pero aún sus indicadores son altos tanto a nivel nacional como provincial, siendo estas tasas un reflejo de las condiciones de vida y calidad de salud de la población en general.

Según datos del Instituto Nacional de Estadística y Censos (INEC), en el Ecuador las proyecciones para el año 1990, los niños menores de 5 años representaban el $38,7 \%$ de la población total nacional y el grupo materno ( mujeres en edad fértil) era el 25.2\%. Estos datos nos revelan condiciones demográficas que nos estarian demostrando las relaciones existentes entre la población materna infantil y la general.

La cobertura de los servicios de salud en el País no abastece las necesidades la población de manera particular en las zonas rurales, por los que eventos como el nacimiento de un niño no cuentan con atención profesional; realizándose en forma empírica un elevado porcentaje aumentando la frecuencia de morbilidad y mortalidad en la madre y el niño.

Esta situación se mantiene en condiciones idénticas en la provincia de Esmeraldas, siendo esta problemática muy sentida por los habitantes de estos lugares por el impacto social, económico y cultural que representa, motivo por el cual se llegó a realizar el presente estudio a través de la cual podríamos identificar las causas evitables de muerte y enfermedad y lo más importante su relación directa con prácticas y creencias comunitarias. De acuerdo a estas creencias puede augurarse que los individuos responsabilizan a hechos sobrenaturales su incapacidad para resolver problemas de salud ya sea por falta de medios económicos, inaccesibilidad a los servicios de salud, creencias y tradiciones mantenidas desde mucho tiempo atrás.

\section{MATERIAL Y MÉTODOS}

El estudio se desarrolló en las comunidades (de negros e indios) de más de 50 familias del Área de salud Borbón de la ciudad de EsmeraldasEcuador, se lo realizó a través de un diseño longitudinal de tipo retrospectivo analítico y comparativo, durante el año 1994. Se tomó encuenta el universo de embarazadas y niños menores de 5 años vivos y muertos de la zona. La muestra definitiva fue de 207 niños menores de 5 años, 83 embarazadas y 7 madres de niños muertos por causas desconocidas.

Las variables recogidas en el presente estudio son edad materna (expresada en años) paridad, 
control del embarazo, atención del parto, finalización del embarazo, estado civil, grupo étnico, controles prenatales, condiciones del nacimiento del niño, actividades de la madre, ingresos familiares, rol de la madre en el contexto familiar y escolaridad, prácticas y creencias en el embarazo, parto y puerperio, cuidado del ombligo, vacunación, enfermedad y muerte.

La información fue obtenida a través de dos tipos de encuesta una materno que contenían preguntas que estaban dirigidas a las mujeres embarazadas y una infantil dirigida a madres de niños menores de 5 años y de niños muertos por causas desconocidas. Entrevistas a informantes claves (personas de las comunidades que habian permanecido en contacto directo con sujetos afectados) y observación directa (permanencia transitoria en cada uno de los centros de salud escogidos para la obtención de la información), las preguntas fueron contestadas a través de la intervención directa entre el entrevistador y los sujetos elegidos, se revisaron las historias de salud de los niños fallecidos. Con todos los datos obtenidos se realizó un análisis estadístico de carácter descriptivo utilizó el programa estadístico EPI.INFO STAL CALC, donde se distribuyó las frecuencias absolutas y porcentajes, así como la presentación gráfica de los resultados más relevantes.

\section{RESULTADOS}

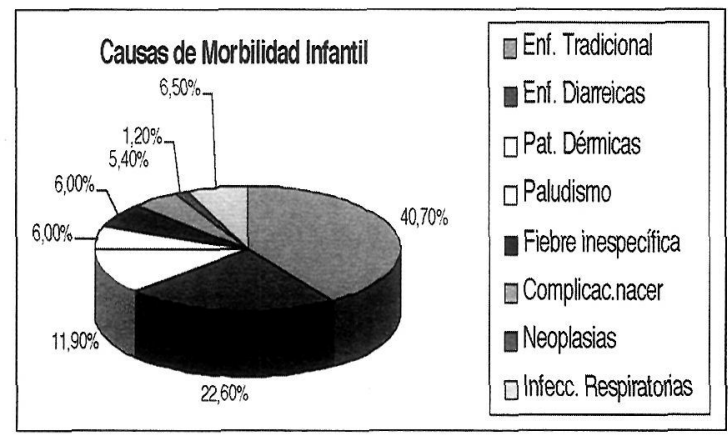

\section{Causas de Morbilidad Materna en el puerperio}
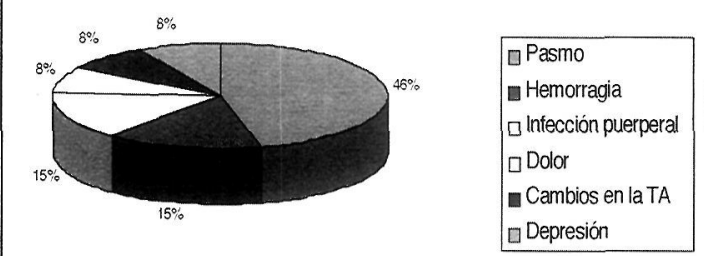

Causas de morbilidad en el embarazo

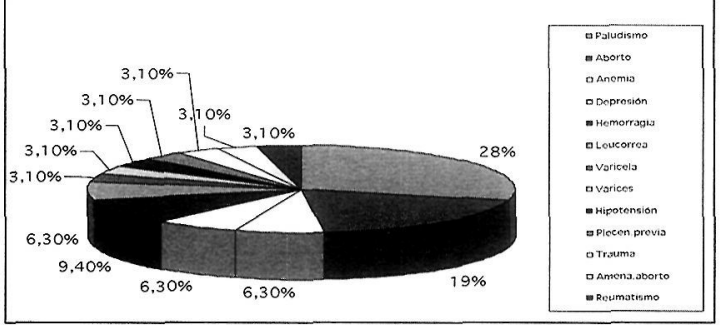

\section{DISCUSIÓN}

Los factores culturales tienen una influencia significativa en la vida de cualquier grupo humano, en la población estudiada hemos llegado a observar que algunas prácticas y creencias comunitarias en determinado momento y con la asociación de otros factores de riesgo pueden llegar a ser nocivos y fatales.

El caso más significativo es la mortalidad infantil en la cual de las 7 muertes ocurridas en ese año, cuatro de ellas fueron atribuidas por las mismas madres a la llamada virtud, implicando la posibilidad de evitar la muerte de estos niños ya que mencionan que sólo personas especiales (brujos) o Dios puede evitar estas muertes, que en muchos caso manifiestan la misma sintomatología de enfermedades como la desnutrición, deshidratación y que pueden llegar a ser evitables.

El riesgo de morbilidad más significativo en el embarazo en estas zonas es la malaria o paludismo, por ser una área endémica y donde la accesibilidad geográfica impide tomar medidas a tiempo para solucionar problemas por situaciones que pueden llegar a ser mortales. 
Hemos llegado a palpar que las llamadas enfermedades tradicionales son de gran importancia para estos pueblos en el proceso salud enfermedad, pues la mayoría de estas personas acuden a solucionar sus problemas de salud donde personas que se hacen llamar curanderos para tratar enfermedades que ellos denominan el ojo, espanto y malaire, antes que a los centros de salud establecidos en la comunidad que en muchos casos estas enfermedades son atribuibles a enfermedades diarreicas y respiratorias.

Otro aspecto importante dentro de nuestro estudio es la manera de cómo curaban el ombligo de los recién nacidos, que consistía en la aplicación de sustancias del medio con el objetivo de atribuirles ciertas facultades especiales que serán de mucha ayuda en el futuro, con el cual estamos viendo la amenaza que representa para la salud del RN, puesto en su mayoría estas sustancias contienen microorganismos que provocarían secuelas graves o mortales para el niño

\section{AGRADECIMIENTO}

Dedicado a todas aquellas mujeres y niños que enfermaron y murieron por causas evitables, al Prorector Jokin Zurutuza y cada uno de los miembros de la Pontificia Universidad Católica del Ecuador con sede en Esmeraldas, al Departamento de Enfermería de la Universidad de Alicante y en especial a Dr. Miguel Richart porque han hecho posible que permitamos presentar la realidad cruda por la que atraviesa nuestro país.

\section{BIBLIOGRAFIA}

1. Boletín Epidemiológico (1993). \# 3. Enero- Marzo.

2. Boletín Epidemiologico de Antioquía (1993). Servicio Nacional de Salud de Antioquía.

3. Manos Unidas (1993). Campaña contra el hambre.

4. Diez principales causas de Mortalidad Infantil (1993). Departamento de Estadística de la Dirección Provincial de Salud del Guayas

5. Ministerio de Salud Pública del Ecuador. Manual de Organización de las áreas de salud.

6. Organización Panamericana de la Salud (1986). Manual sobre enfoque de Riesgo en la Atención Materno infantil. México.

7. Organización Panamericana de la Salud (1984). Salud Materno Infantil y atención primaria en las Américas. México.

8. Baladilline (1990), P. Entre lo Mágico y la Natural. 2. a ed. Abya-yala.

9. Canales, F., Otros (1986). Metodología de la Investigación. ed. Limusa. México.

10. Holl, J., Otros (1990). Enfermería en Salud Comunitaria. Un enfoque de Sistemas. Ed. OPS. Washington.

11. Meneghello, RJ. (1985) Pediatría. 3." ed. Mediterráneo. Chile.

12. Naranjo, P. (1991). Plan Nacional de Salud 1991-2000. ed. OMS. Ecuador.

13. Niswander, K. (1985). Manual de Obstetricia. 2." ed. Salvat. España.

14. Ramos, G. (1989). Alto Riesgo Obstétrico. ed. UCE. Quito.

15. Ramirez, M. (1984). Folklore de la zona Norte Esmeraldeña. ed. Banco Central del Ecuador. Quito.

16. Reeder, S., otros. (1988). Enfermería Materno Infantil. 5.a ed. OPS. México.

17. SAvola, R. (1988). El negro en la Historia del Ecuador y el Sur de Colombia. ed. Centro Cultural Afroecuatoriano. Quito.

18. SPEIser, S. (1987), Religiosidad Popular Afroesmeraldeña. Ed. Vicariato Apostólico de Esmeraldas. Esmeraldas.

19. Juanita y Glovanino. (1992). Análisis de Mortalidad: Un instrumento de Servicio.

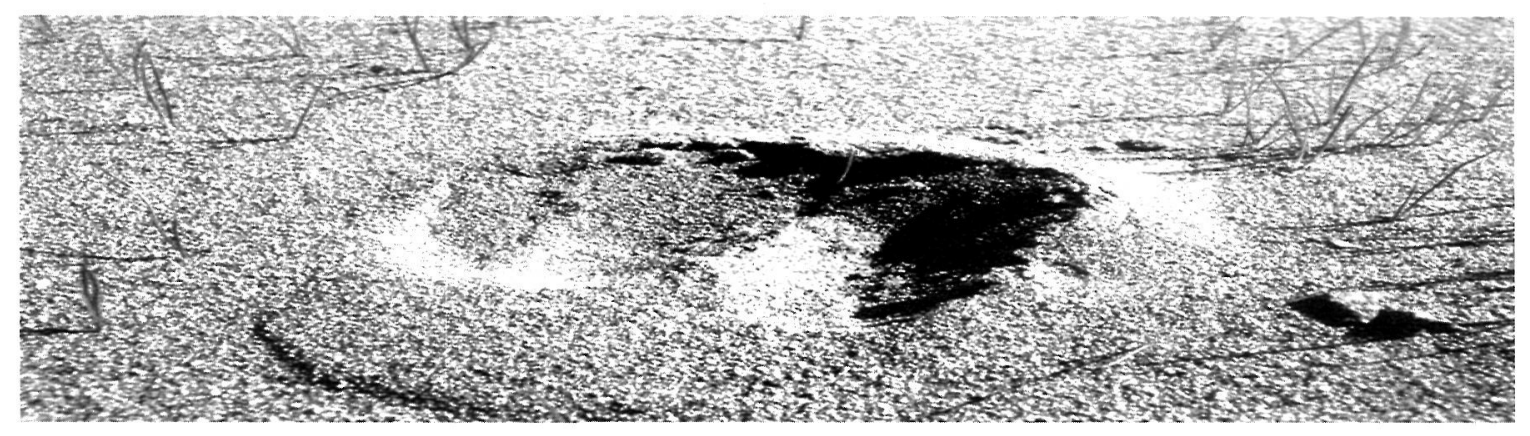

